DOI: $10.1002 /(($ please add manuscript number $))$

Article type: Communication

\title{
Monitoring Implantable Immunoisolation Devices with Intrinsic Fluorescence of Genipin
}

Edorta Santos-Vizcaino, Henry Haley, Ainhoa Gonzalez-Pujana, Gorka Orive, Rosa Maria Hernandez, Gary D. Luker*, Jose Luis Pedraz*

Dr. E. Santos-Vizcaino, A. Gonzalez-Pujana, Dr. G. Orive, Prof. R.M. Hernandez, Prof. J.L. Pedraz

NanoBioCel Group, Laboratory of Pharmaceutics, School of Pharmacy, University of the Basque Country (UPV/EHU),

Vitoria-Gasteiz, 01006, Spain.

Dr. E. Santos-Vizcaino, A. Gonzalez-Pujana, Dr. G. Orive, Prof. R.M. Hernandez, Prof. J.L. Pedraz

Biomedical Research Networking Centre in Bioengineering, Biomaterials and Nanomedicine (CIBER-BBN),

Vitoria-Gasteiz, 01006, Spain.

H. Haley, Prof. G.D. Luker

Department of Radiology, Center for Molecular Imaging, University of Michigan Medical School, Ann Arbor, MI 48109, USA.

G.D. Luker

Department of Biomedical Engineering, University of Michigan Medical School, Ann Arbor, MI 48109, USA.

G.D. Luker

Department of Microbiology and Immunology, University of Michigan Medical School, Ann Arbor, MI 48109, USA.

This is the author manuscript accepted for publication and has undergone full peer review but has not been through the copyediting, typesetting, pagination and proofreading process, which may lead to differences between this version and the Version of Record. Please cite this article as doi: 10.1002/jbio.201800170

This article is protected by copyright. All rights reserved. 


\section{WILEY-VCH}

E-mail:

Prof. J.L. Pedraz: joseluis.pedraz@ehu.eus

Prof. G.D. Luker: gluker@med.umich.edu

Keywords: Genipin, quantitative imaging, biosafety, immunoisolation devices, hydrogels

Imaging of implanted hydrogel-based biosystems usually requires indirect labelling of the vehicle or cargo, adding complexity and potential risk of altering functionality. Here, for the first time, it is reported that incorporating genipin into the design of immunoisolation devices can be harnessed for in vivo imaging. Using cell-compatible in situ cross-linking reactions, a fast, efficient and non-cytotoxic procedure is shown to maximize fluorescence of microcapsules. Moreover, genipin is validated as a quantitative imaging probe by injecting increasing doses of microcapsules in the subcutaneous space of mice, obtaining strong, stable fluorescence with good linearity of signal to microcapsule dose over several weeks. This allows immediate assessment of the actual injected dose and monitoring of its position over time, thereby significantly enhancing the efficacy and biosafety of the therapy. These outcomes may facilitate clinical translation and optimize medical applications of multiple hydrogel-based biotechnologies.

This article is protected by copyright. All rights reserved. 
Designing hydrogel technologies for detection by imaging frequently requires modifying the hydrogel itself ${ }^{[1,2]}$ or the cargo, ${ }^{[3]}$ adding complexity to overall design and potentially altering mechanical and functional characteristics. ${ }^{[4]}$ We sought to overcome these challenges by incorporating a biomaterial with inherent imaging properties into device design. While applicable broadly to other areas in biotechnology, in the present study we focus on immunoisolation devices consisting of alginate microspheres coated with a semipermeable membrane formed by polycations (usually poly-L-Lysine or poly-L-ornithine). The membrane protects the encapsulated cell content against immune cell and antibody mediated host's rejection, while allowing the inward diffusion of nutrients and oxygen, and the release of bioactive compounds into the surrounding tissue. This approach currently is one of the leading strategies to deliver bioactive molecules from immobilized allo- or xenogenic cells. ${ }^{[5}$, 6] To date, remarkable outcomes have been obtained in clinical trials for the treatment of chronic diseases such as diabetes ${ }^{[7,8]}$ or cancer. $^{[9,10]}$ However, development of a suitable noninvasive visualization strategy that provides relevant information about implanted microcapsules becomes crucial to make the definitive leap to the clinic. ${ }^{[11-13]}$ 


\section{WILEY-VCH}

In this sense, one of the main problems lies in the impossibility to ensure the correct administration of the intended dose. This is because, to the best of our knowledge, to date there is no tool to immediately assess (quantitatively) the actual injected dose and then monitor its position and stability for long periods of time (maintaining a reliable, precise and stable signal/dose relation). Alternative imaging strategies, such as reporter genes based on luciferase or fluorescence proteins, provide poor information immediately after injection, since the hypoxic stress suffered by encapsulated cells during the first days post-implantation makes the emitted signal unreliable. ${ }^{[14-16]}$

Genipin is a natural compound with dual properties that uniquely meet the demand for a hydrogel technology detectable through imaging. Strikingly, while benefits of genipin as a cross-linker are increasingly appreciated due to its superior biocompatibility, use of natural fluorescence from this material has generally been overlooked or considered only anecdotally. ${ }^{[17-20]}$ Contrarily, in the nano-scale, genipin-cross-linked globin-PEI nanoparticles $^{[21]}$ and genipin cross-linked ovalbumin protein nanoparticles ${ }^{[22]}$ have been recently reported as suitable for in vivo imaging. However, described methods are far from being applicable to higher scaled cell-laden hydrogels for cell therapies. Indeed, to the best of our knowledge, no one has achieved a cytocompatible in situ cross-linking of genipin with optimal brightness for in vivo imaging of hydrogel technologies. 


\section{WILEY-VCH}

Here, for the first time, we harness the natural fluorescence of genipin to produce bright, quantitative and stable fluorescence for in vivo imaging of cell-laden hydrogel systems. Following excitation with red light, genipin emits far-red fluorescence with a broad tail extending into the near infra-red spectrum (Figure S1). ${ }^{[21]}$ These wavelengths of light are highly favorable for in vivo imaging studies, particularly for hydrogels designed for implantation in superficial sites such as subcutaneous, subdermal, or intra-ocular. ${ }^{[23]}$ Our results obtained with alginate-poly-L-Lysine immunoisolation devices show that fluorescence from genipin meets the need for quantitative imaging of implanted biomaterials. By incorporating a biomaterial with inherent imaging properties into device design, this groundbreaking advance will facilitate clinical translation of a wide range of hydrogel technologies for cell-based therapies and tissue engineering.

To obtain a good signal to noise ratio for imaging in vivo, maximizing the fluorescence becomes indispensable. Thus, we first optimized the cross-linking procedure of genipin while maintaining cell viability using D1 mesenchymal stem cells from Balb/c mice genetically engineered to secrete human erythropoietin (D1-MSC-hEPO). We subjected cells encapsulated in alginate-poly-L-Lysine (AP) to different combinations of genipin concentrations $(0.001 \%, 0.01 \%$ and $0.1 \%)$ and exposure times $(5,15,30$ and $60 \mathrm{~min}$ at room temperature) (Figure 1a). For each grouping, we recorded fluorescence intensity of the microspheres (Ex: $590 \mathrm{~nm}$; Em: $630 \mathrm{~nm}$ ) and viability of encapsulated cells. We plotted results as a function of these two variables (Figure $1 \mathbf{b}, \mathbf{c})$. As a control group, we used cells immobilized in non-cross-linked AP microcapsules. Based on these screening assays, we 


\section{WILEY-VCH}

chose the best four conditions where we obtained the highest fluorescence intensities with statistically non-significant losses in viability compared with the AP control group (Figure 1b,c, white points): $0.01 \% \& 30 \min <0.01 \% \& 60 \min <0.1 \% \& 5 \min <0.1 \% \& 15 \min (p$ $<0.001$ for all comparisons) (Figure S2).

After examining these four protocols in detail (Figure 1d), we chose $0.1 \%$ genipin and 5 min exposure time as the optimum conditions. Despite rendering lower fluorescence intensity than the $0.1 \%$ \& 15 min combination, the latter showed more internal fluorescence (cross-linked cells), so we preferred shorter exposure times to accelerate the coating process and allow greater flexibility and safety margin with respect to cell viability. Indeed, most studies to date use longer exposure times to genipin (from $30 \mathrm{~min}$ to 24 hours), ${ }^{[17,20,24]}$ which may limit dramatically the applicability of this cross-linking agent for cell microencapsulation purposes. Thus, our genipin-meditated cross-linking procedure is fast, efficient and non-cytotoxic, even in the presence of cells.

With the aim of maximizing fluorescence signal of the microcapsules, we next added a second coating of poly-L-Lysine (PLL) cross-linked with genipin, following the same protocol described above $(0.1 \%$ genipin and 5 min exposure). This process produced genipin-crosslinked double poly-L-Lysine membranes (GDP) (see 3D morphology in Video S1). As intermediate control groups, we also tested AP microcapsules cross-linked with genipin (APG), AP with a second PLL coating (APP) and APG microcapsules with an additional covering of PLL (APGP) (Figure 2a). All genipin-containing groups developed maximum 


\section{WILEY-VCH}

fluorescence values after 72 - 96 hour periods of incubation (cell culture conditions, after exposure to genipin) (Figure 2b), following a first-order reaction (Figure S3). Interestingly, we could clearly observe that GDP microcapsules emitted 6-fold higher fluorescence intensity than APG and APGP counterparts ( $p<0.01$ and 0.001, respectively) (Figure 2c,d), probably due to increased availability of PLL for cross-linking. This gain in fluorescence is essential for maximizing the signal to noise ratio for imaging in vivo. In addition, we easily can augment fluorescence intensity by producing smaller size microcapsules. Here, we obtained a 2.5 fold-increase in fluorescence intensity by just reducing diameter of microcapsules by $33 \%$ $(p<0.01)$ (Figure S4).

To exclude any negative effects on cell integrity and function as a consequence of incorporating so many variations in microcapsule design, we tested different control groups to detect possible problems at any step of the formulation. The protocol for producing GDP microcapsules reliably maintained viability of encapsulated cells (Figure 2e,f and Video S2) and the capacity of immobilized cells to secrete high rates of therapeutic product, in this case human erythropoietin (hEPO) (Figure 2g). All these assays showed no significant differences with respect to the AP control group.

Then, we tested performance of GDP microcapsules as a monitoring system for in vivo imaging. For such aim, we implanted 50, 100 or $200 \mu \mathrm{L}$ of GDP microcapsules (Figure 3a) into the subcutaneous space of NSG mice. Images obtained with $570 \mathrm{~nm}$ excitation and a 620/20 emission filter exhibited a strong signal with excellent signal to noise ratio for all 


\section{WILEY-VCH}

injections (Figure 3b). Importantly, we also achieved a good linearity of fluorescence response to microcapsule dose $\left(\mathrm{R}^{2}=0.9971\right)$ (Figure 3c). We next monitored fluorescence of genipin at days 1, 14, 21 and 35 to validate robustness and long-term stability of this noninvasive visualization strategy (Figure 3d-g). The signal decreased significantly in $50 \mu \mathrm{L}$ dose by day $21(p<0.05)$ but remained relatively stable until day 35. Conversely, 100 and $200 \mu \mathrm{L}$ doses showed strong signals that did not differ from the initial point throughout the experiment (Figure 3h). Because we injected capsules in PBS, fluorescence signal appears modestly less intense and more diffuse on the pseudocolor images of day 1. As PBS resorbs, capsules become slightly more localized and fluorescence signal is clearer. Anyhow, differences with respect to day 1 are not significative in any case $(p>0.05)$. As a control for stability of fluorescence, we monitored non-implanted GDP microcapsules from the same batch in parallel, showing non-significant differences in signal throughout the experiment (Figure 4a).

We further analyzed imaging data to validate genipin as a quantitative imaging probe for implanted biomaterials. We first calculated the correlation between administered dose and measured fluorescence signal. A scatter plot of dose versus radiant efficiency and analysis of the linear regression confirmed the linear correlation $(p<0.001)$ and the significance of the slope obtained from the equation $(p<0.001)$. However, the goodness of fit demonstrated that only $66.14 \%$ of the results could be explained by this equation (Figure $4 \mathbf{b}$ ). Taking into account the high R-squared observed when we used mean values, instead of individual values, for linear regression (Figure 3c), we hypothesized that low precision arisen from either 


\section{WILEY-VCH}

instrument or human error should be behind the poor fitting. In addition, from mean and SD values of the background signal, we estimated the lower limit of detection (LLD) and the lower limit of quantification (LLQ), obtaining doses equivalent to $18.6 \mu \mathrm{L}$ and $59.6 \mu \mathrm{L}$ for each of them respectively (Figure $\mathbf{4 b}$ ). This means that the significant signal decay observed for $50 \mu \mathrm{L}$ dose over time (Figure $\mathbf{3 h}$ ) should be considered as non-reliable.

Considering the proven stability of the fluorescence signal, we calculated the variability of measurements taken at different time points for each dose as an indicator of the instrument error (repeatability). The lowest dose of $50 \mu \mathrm{L}$ produced a significantly higher coefficient of variation $(\mathrm{CV})(46.5 \%)$, whereas 100 and $200 \mu \mathrm{L}$ doses presented $25.7 \%$ and $20.8 \%$ respectively (Figure 4c). These results are comprehensible if we take into account $50 \mu \mathrm{L}$ dose is below the LLQ. Consequently, the high variability of the lowest dose may be the main determinant of the poor goodness of fit. By isolating this variability, we also determined the human error in the injections of the microcapsules (i.e. dose preparing and administration of microcapsules). Thus, in the present study we estimated a CV of $39.8 \%, 50.8 \%$ and $22.2 \%$ for 50, 100 and $200 \mu \mathrm{L}$ doses respectively (Figure 4d). On the other hand, linear regression analysis of the scatter plot for expected versus measured values confirmed the slope of the equation was equal to $1(p<0.001)$ and the intercept equal to 0 (Figure 4e). Indeed, the accuracy of the results with all doses was close to the $100 \%$ (Figure $4 \mathbf{f}$ ).

These results reveal that genipin-mediated fluorescence in GDP microcapsules is detectable even at low doses $(50 \mu \mathrm{L}$ is below the usually administered dose $)(\mathrm{LLD}=18.6 \mu \mathrm{L})$ and reliably quantifiable from $100 \mu \mathrm{L}$ and higher $(\mathrm{LLQ}=59.6 \mu \mathrm{L})$. The ability to image GDP 


\section{WILEY-VCH}

fluorescence provides a powerful tool to immediately assess the quality of injection and then monitor stability of microcapsule signal over time with more than acceptable variability of measurements with usually administered doses $(>200 \mu \mathrm{L})$. Human errors we made and quantified in the present study are common in standard practice in the field of cell microencapsulation but undetectable without the technology described here. Therefore, GDP microcapsules represent a valuable tool to ensure correct administration of the intended dose and improve efficacy and biosafety of cell encapsulation therapies in the clinical routine.

Unlike hydrogels with homogeneous cross-linking throughout their whole volume, GDP microcapsules, as cell-laden alginate microspheres with fluorescence limited to the external membranes (few microns), represent a demanding model for genipin-mediated in vivo imaging. This means that as capsule diameter increases, fluorescence signal related to a particular administered dose diminishes. Therefore, this visualization strategy will work much better when using particle sizes with high surface-area-to-volume ratio. Future studies will also reveal the feasibility of GDP capsule design for other usually used routes of administration, including intraperitoneal, intravitreal or intracranial.

In summary, we present a multidisciplinary approach to develop implantable biosystems based on hydrogels with intrinsic fluorescence for in vivo imaging. In particular, we have shown that the use of genipin, an increasingly accepted cross-linker, functions as an excellent quantitative imaging probe to be included in the design of immunoisolation devices. Through this strategy, we have managed to visualize not only the location of the implanted 


\section{WILEY-VCH}

microcapsules, but also to evaluate the actual injected dose, which may improve significantly the efficacy and biosafety of the therapy. As fluorescence imaging systems are gradually implemented in clinical practice, we believe these outcomes will have direct applicability to advance design of multiple hydrogel-based biotechnologies, including drug and cell delivery systems, vaccines or biosensors.

\section{Experimental Section}

All materials and methods used in this study are thoroughly detailed in the Supporting Information.

The University of Michigan IACUC approved all animal procedures.

\section{Supporting Information}

Supporting Information is available from the Wiley Online Library or from the author.

\section{Acknowledgements}

This project was supported by the Basque Government (Consolidated Groups, IT-907-16) and United States of America grants from the National Institutes of Health (R01CA196018 and U01CA210152). Ainhoa Gonzalez-Pujana thanks the Basque Government (Department of 


\section{WILEY-VCH}

Education, Universities and Research) for the PhD grant. Authors thank for the technical and human support provided by SGIker of UPV/EHU, European funding (ERDF and ESF) and ICTS "NANBIOSIS" (Drug Formulation Unit, U10) of the CIBER-BBN at the University of Basque Country UPV/EHU in Vitoria-Gasteiz. We also thank Dr. Felipe Prosper at the University Clinic of Navarra (CUN) for his assistance on the development of the lentiviral vector pSIN-EF2-Epo-Pur. Edorta Santos-Vizcaino thanks Ricardo Andrade and Pedro Guerrero for their technical support and invaluable advice. Both Gary D. Luker and Jose Luis Pedraz are corresponding authors.

Received: ((will be filled in by the editorial staff))

Revised: ((will be filled in by the editorial staff)) Published online: ((will be filled in by the editorial staff))

\section{References}

[1] J. Liang, X. Dong, C. Wei, D. Kong, T. Liu, F. Lv, R.S.C. Adv. 2017, 7, 6501.

[2] A. Berdichevski, H. Simaan Yameen, H. Dafni, M. Neeman, D. Seliktar, Proc. Natl. Acad. Sci. U. S. A. 2015, 112, 5147.

[3] B. P. Barnett, A. Arepally, M. Stuber, D. R. Arifin, D. L. Kraitchman, J. W. Bulte, Nat. Protoc. 2011, 6, 1142.

[4] A. A. Appel, M. A. Anastasio, J. C. Larson, E. M. Brey, Biomaterials. 2013, 34, 6615. 


\section{WILEY-VCH}

[5] G. Orive, E. Santos, D. Poncelet, R. M. Hernandez, J. L. Pedraz, L. U. Wahlberg, P. de Vos, D. Emerich, Trends Pharmacol. Sci. 2015, 36.

[6] A. J. Vegas, O. Veiseh, M. Gurtler, J. R. Millman, F. W. Pagliuca, A. R. Bader, J. C. Doloff, J. Li, M. Chen, K. Olejnik, H. H. Tam, S. Jhunjhunwala, E. Langan, S. ArestaDasilva, S. Gandham, J. J. McGarrigle, M. A. Bochenek, J. Hollister-Lock, J. Oberholzer, D. L. Greiner, G. C. Weir, D. A. Melton, R. Langer, D. G. Anderson, Nat. Med. 2016, 22, 306.

[7] G. Basta, P. Montanucci, G. Luca, C. Boselli, G. Noya, B. Barbaro, M. Qi, K. P. Kinzer, J. Oberholzer, R. Calafiore, Diabetes Care. 2011, 34, 2406.

[8] T. Desai, L. D. Shea, Nat. Rev. Drug Discov. 2017, 16, 338.

[9] M. Löhr, A. Hoffmeyer, J. Kröger, M. Freund, J. Hain, A. Holle, P. Karle, W. T. Knöfel, S. Liebe, P. Müller, H. Nizze, M. Renner, R. M. Saller, T. Wagner, K. Hauenstein, W. H. Günzburg, B. Salmons, Lancet. 2001, 357, 1591.

[10] J. M. Löhr, S. L. Haas, J. C. Kröger, H. M. Friess, R. Höft, P. E. Goretzki, C. Peschel, M. Schweigert, B. Salmons, W. H. Gunzburg, Pharmaceutics. 2014, 6, 447.

[11] N. G. Kooreman, J. D. Ransohoff, J. C. Wu, Nat. Mater. 2014, 13, 106.

[12] E. Santos, J. L. Pedraz, R. M. Hernández, G. Orive, J. Control. Release. 2013, 170, 1.

[13] G. A. Paredes-Juarez, P. de Vos, J. W. Bulte, Expert. Rev. Precis. Med. Drug. Dev. 2017, 2,57 .

[14] R. Catena, E. Santos, G. Orive, R. M. Hernández, J. L. Pedraz, A. Calvo, J. Control. Release. 2010, 146, 93. 


\section{WILEY-VCH}

[15] E. Santos, L. Larzabal, A. Calvo, G. Orive, J. L. Pedraz, R. M. Hernandez, Biomaterials. 2012, 34, 1442.

[16] A. Goren, N. Dahan, E. Goren, L. Baruch, M. Machluf, FASEB J. 2010, 24, 22.

[17] A. Paul, G. Chen, A. Khan, V. T. Rao, D. Shum-Tim, S. Prakash, Cell Transplant. 2012, 21(12), 2735.

[18] H. Chen, W. Ouyang, B. Lawuyi, S. Prakash, Biomacromolecules. 2006, 7(7), 2091.

[19] H. Chen, W. Ouyang, B. Lawuyi, C. Martoni, S. Prakash, , J. Biomed. Mater. Res. A. 2005, 75(4), 917.

[20] A. L. Hillberg, K. Kathirgamanathan, J. B. Lam, L. Y. Law, O. Garkavenko, R. B. Elliott, J. Biomed. Mater. Res. B. Appl. Biomater. 2012, 101, 258.

[21] Y. Zhang, L. Mao, J. Liu, T. Liu, Mat. Sci. Eng. C. 2017, 71, 17.

[22] X. Dong, Z. Sun, J. Liang, H. Wang, D. Zhu, X. Leng, C. Wang, D. Kong, F. Lv, Nanomedicine. 2018, 14(4), 1087.

[23] G. D. Luker, K. E. Luker, J. Nucl. Med. 2008, 49(1), 1.

[24] A. Paul, A. Cantor, D. Shum-Tim, S. Prakash, Mol. Biotechnol. 2011, 48(2), 116. 


\section{WILEY-VCH}
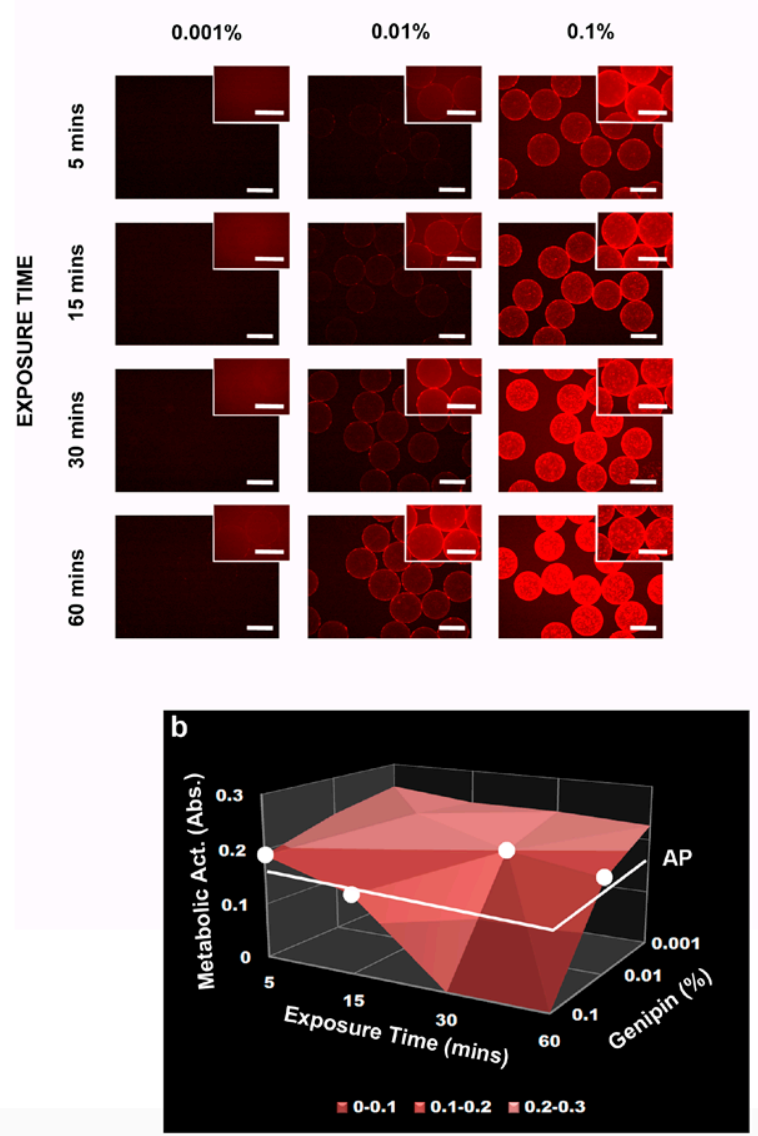

d
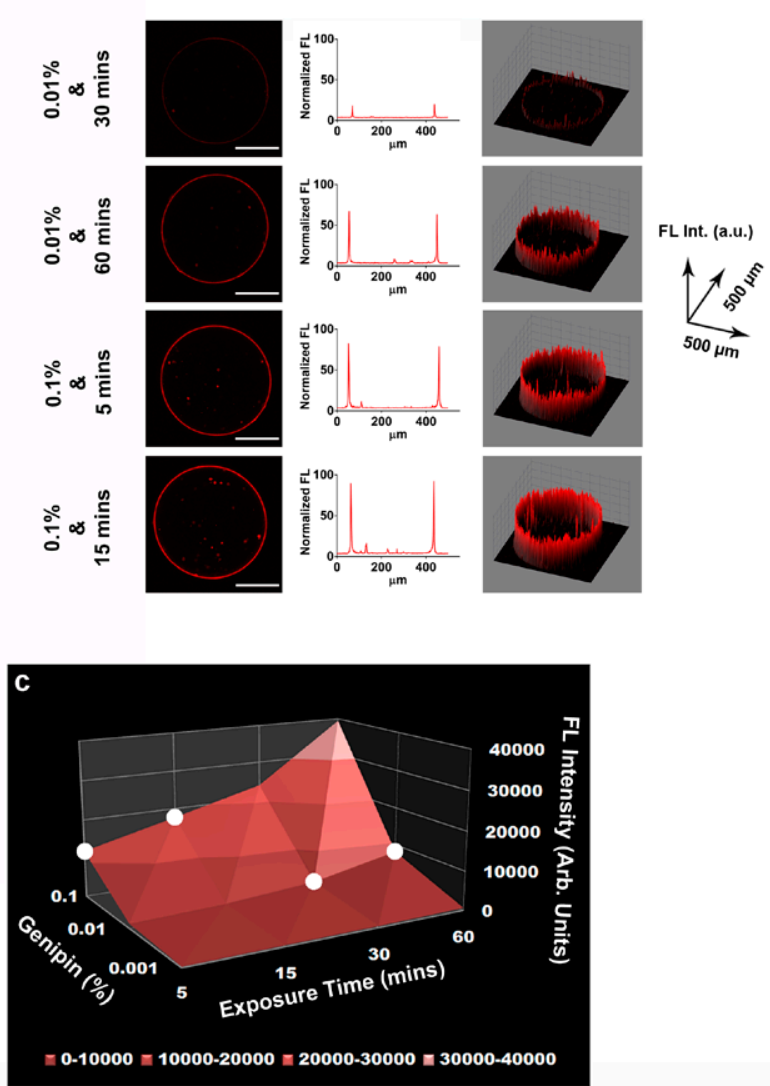

Figure 1. Optimization of genipin cross-linking procedure in cell-laden microcapsules. D1-MSC-hEPO enclosed within AP microcapsules were subjected to increasing genipin concentrations $(0.001 \%, 0.01 \%$ and $0.1 \%)$ and exposure times $(5,15,30$ and $60 \mathrm{~min})$ to find the optimum conditions. a, Representative epi-fluorescence micrographs of each concentration and time combination. Scale-bar, $400 \mu \mathrm{m}$. b, Three-dimensional plot representing mean metabolic activity values of encapsulated cells in function of different genipin concentrations and exposure times. The white line indicates the mean value from noncross-linked AP control group. c, Three-dimensional plot representing mean fluorescence intensities of AP microcapsules in function of different genipin concentrations and exposure times. b,c, Color scale from light red to dark red denotes highest and lowest values, respectively. The white points depict the highest fluorescence signal intensities obtained without affecting cell viability (non-significant differences against cells encapsulated in noncross-linked AP microcapsules, $p>0.05$ ). Statistical analysis: one-way ANOVA with 


\section{WILEY-VCH}

Bonferroni multiple comparison correction, $n=5$ samples for each assay. d, Analysis of the conditions selected in $(\mathbf{b}, \mathbf{c})$ by means of confocal fluorescence microscopy. From left to right: representative confocal fluorescence micrographs, profile lines and 3D surface plots of the fluorescence signal distribution and intensity from the equatorial section of microcapsules. Scale bar, $200 \mu \mathrm{m}$. FL, fluorescence.

a
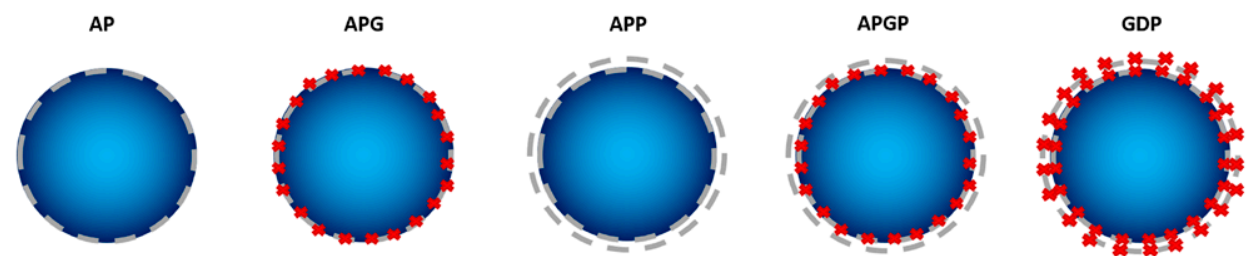

Alginate matrix

Poly-L-lysine coating

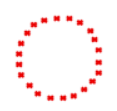

Genipin cross-linking

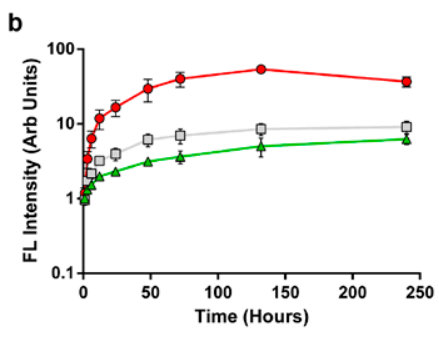

$\triangle$ APG $\square$ APGP $\rightarrow$ GDP
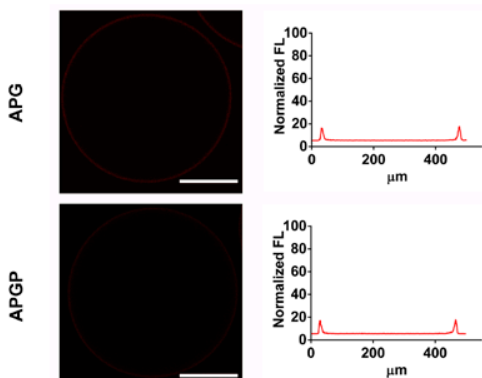

ㅇํํ
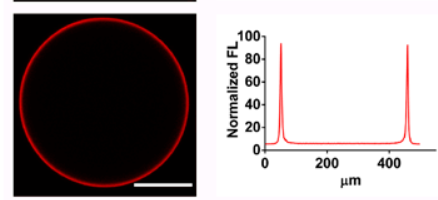
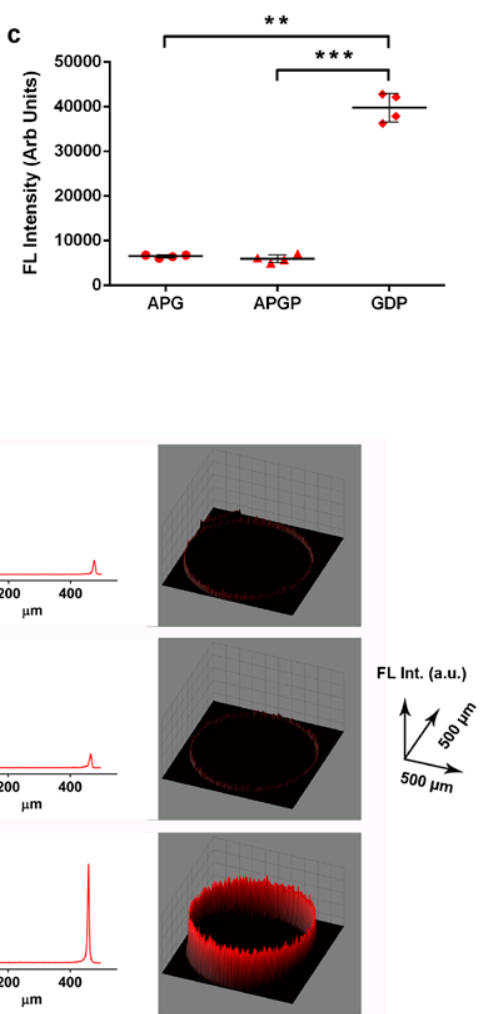

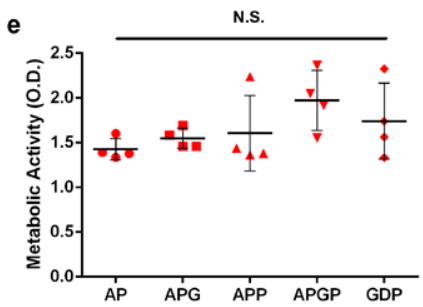

f
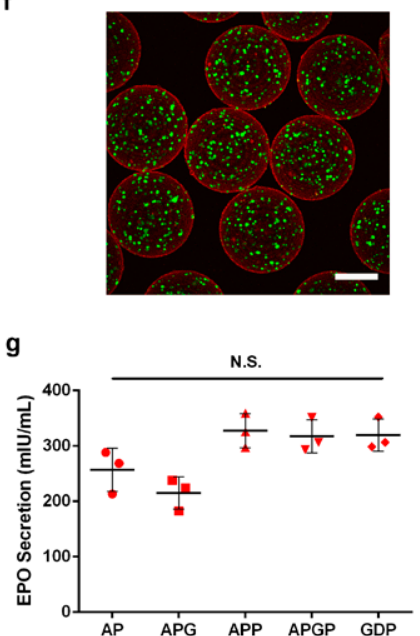

Figure 2. GDP microcapsules maximize the fluorescence of genipin while preserving cell viability and function. a, Eschematic depiction showing GDP microcapsules and all the intermediate control groups assayed in the study. $\mathbf{b}$, Reaction kinetics of genipin fluorescence development (Em: $590 \mathrm{~nm}$; Ex: $630 \mathrm{~nm})(n=4$ samples per group). Error bars, mean \pm SD. c, 


\section{WILEY-VCH}

Fluorescence intensity of microcapsules $96 \mathrm{~h}$ after encapsulation ( $n=4$ samples per group). Error bars, mean $\pm \mathrm{SD}$. ${ }^{* *}, p<0.01$; ${ }^{* *}, p<0.001$; One-way ANOVA with Tamhane multiple comparison correction. d, Confocal fluorescence analysis of microcapsules. From left to right: representative confocal fluorescence micrographs, profile lines and 3D surface plots of the fluorescence signal distribution and intensity from the equatorial section of microcapsules. Scale bar, $200 \mu \mathrm{m}$. e, Metabolic activity of encapsulated D1-MSC-hEPO cells ( $n=4$ samples per group) $96 \mathrm{~h}$ after encapsulation. Error bars, mean \pm SD. N.S. specifies nonsignificant differences against the AP group, $p>0.05$; One-way ANOVA with Bonferroni multiple comparison correction. Mann-Whitney $U$ test was used with APP group (non-normal distribution). f, Representative confocal fluorescence image of cells encapsulated in GDP microcapsules and probed with LIVE/DEAD viability kit (Green, living cells; Red, dead cells) 14 days after encapsulation. g, hEPO secretion of immobilized D1-MSC-hEPO cells ( $n$ $=3$ samples per group in duplicate) $96 \mathrm{~h}$ after encapsulation. Error bars, mean \pm SD. N.S. indicates non-significant differences against the AP group, $p>0.05$; One-way ANOVA with Bonferroni multiple comparison correction. FL, fluorescence.

b

a

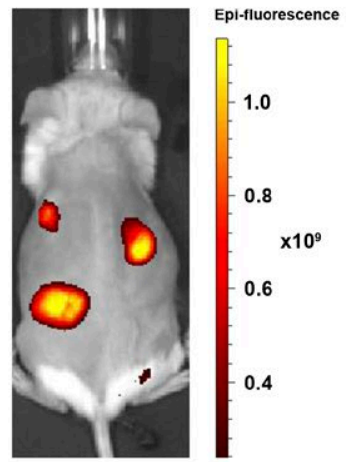

C

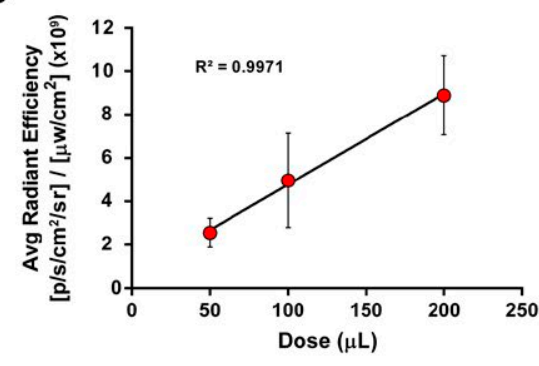

d
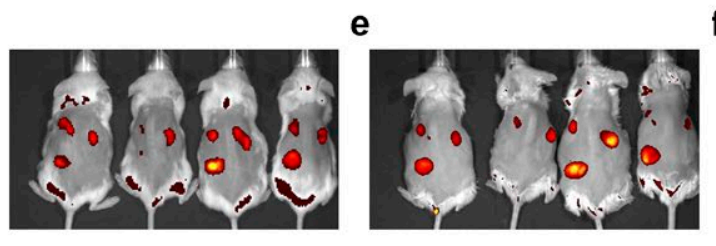

f
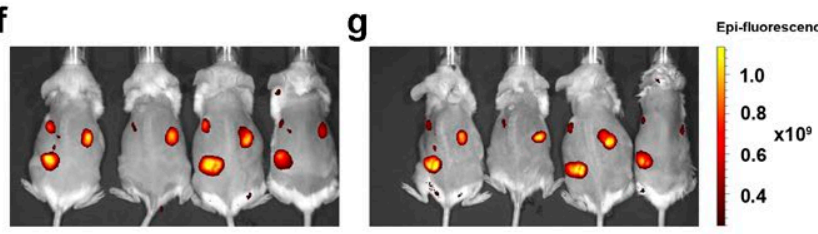

h

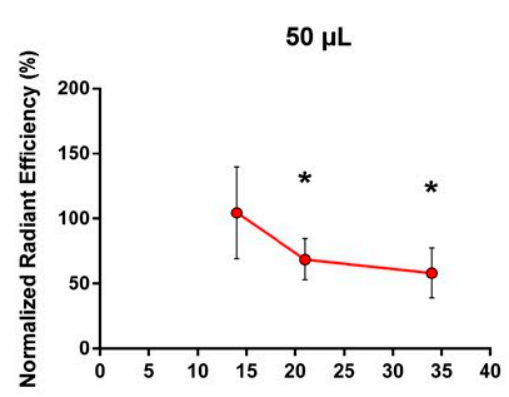

$100 \mu \mathrm{L}$

$200 \mu \mathrm{L}$
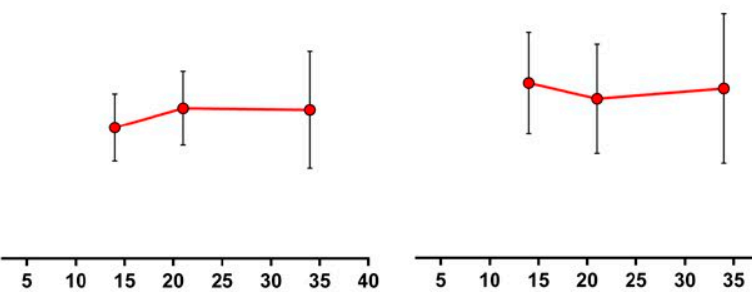

Time (Days) 
Figure 3. GDP microcapsules maintain dose-dependent fluorescence over 35 days in vivo. a. White light image of 50,100 and $200 \mu \mathrm{L}$ of GDP microcapsules in $1.5 \mathrm{~mL}$ microcentrifuge tubes. b. Representative image of a mouse 21 days after injection of GDP microcapsules. We imaged fluorescence from the microcapsules with $570 \mathrm{~nm}$ excitation and $620 \mathrm{~nm}$ emission. Scale bar denotes range of photons displayed on a pseudocolor scale with yellow and dark red denoting highest and lowest values, respectively. c, Graph displays dosedependent response of average radiant efficiency for GDP microcapsules. Error bars, mean \pm $\mathrm{SD}$ ( $\mathrm{n}=4$ per condition) d-g. Panels show representative fluorescence images of mice $1,14,21$ and 35 days after subcutaneous injection of microcapsules. We used the same pseudocolor scale from panel (b) to display fluorescence. h. Graph shows fluorescence of GDP microcapsules remained relatively constant over 35 days. Data are normalized to day 1 images. Error bars, mean $\pm \mathrm{SD} .{ }^{*}, p<0.05$; Paired, two-tailed $t$-test, $n=4$ mice per dose. 


\section{WILEY-VCH}

a

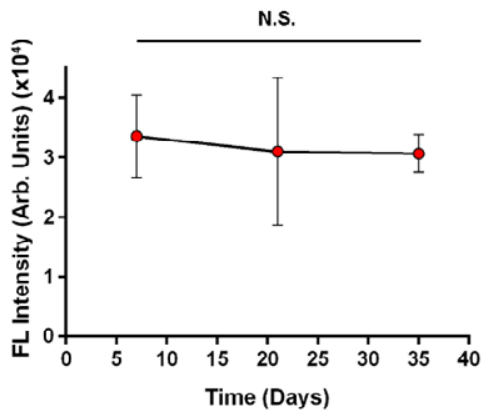

C

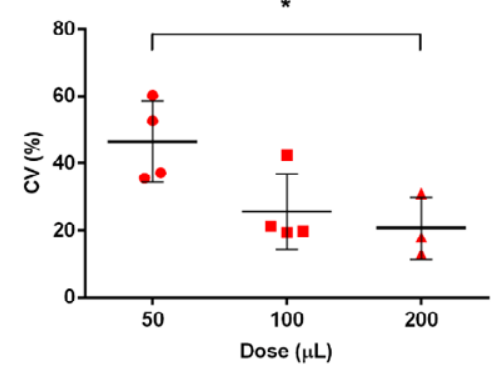

e

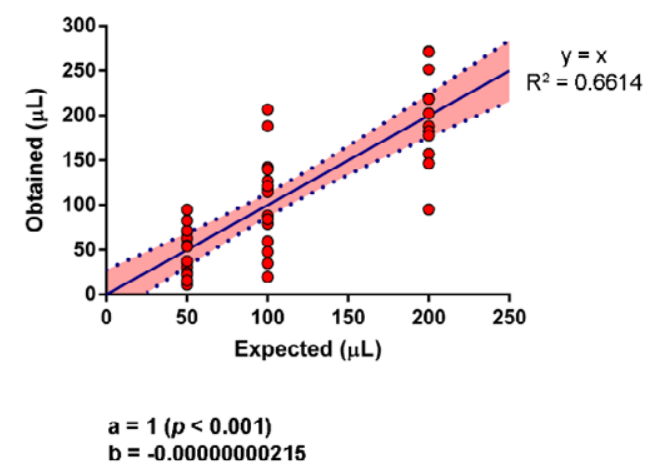

b

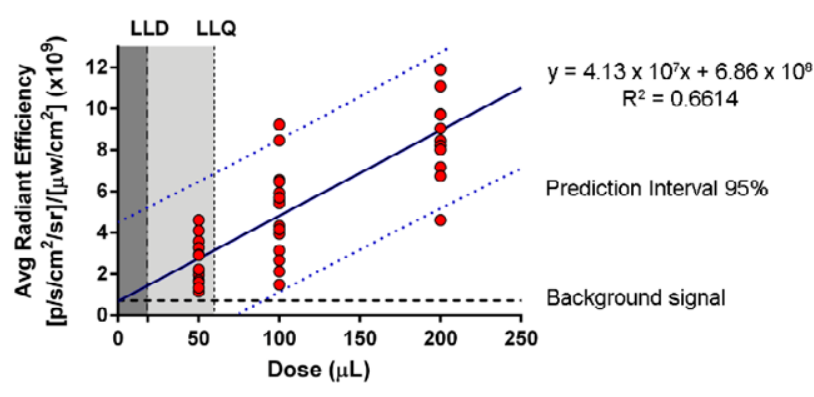

$a=41,339,506(p<0.001)$ $b=685,622,093$

d

\begin{tabular}{lccc}
\hline Human error & $\mathbf{5 0}$ & $\mathbf{1 0 0}$ & $\mathbf{2 0 0}$ \\
\hline Mouse 1 Dose $(\boldsymbol{\mu L})$ & 65.8 & 99.9 & 171.1 \\
Mouse 2 Dose $(\boldsymbol{L L})$ & 30.3 & 104.9 & \\
Mouse 3 Dose $(\boldsymbol{\mu L})$ & 62.0 & 169.3 & 249.1 \\
Mouse 4 Dose $(\boldsymbol{\mu L})$ & 32.2 & 40.5 & 174.9 \\
\hline \hline CV $(\%)$ & 39.8 & 50.8 & 22.2 \\
\hline
\end{tabular}

Figure 4. Analysis of obtained imaging data to validate genipin as a quantitative imaging probe. a. We monitored non-administered GDP microcapsules in parallel throughout experiment. Error bars, mean \pm SD. N.S., non-significant $(p>0.05)$; Paired, two-tailed $t$-test, $n$ $=5$ independent experiments per time-point. b. Scatter plot of the Dose vs Radiant Efficiency showing the linear regression equation with 95\% prediction interval. Each dot in the plot represents an individual measurement for each mouse, time point and dose. The linear correlation was confirmed by ANOVA $(p<0.001)$. The significance of the equation slope was statistically verified by means of the linear regression $t$-test. LLD, lower limit of detection. LLQ, lower limit of quantification. c. Variability of measurements taken at different time points for each dose (repeatability or instrument error). Error bars, mean $\pm \mathrm{SD}$. $*, p<0.05$; One-way ANOVA with Bonferroni multiple comparison correction, $n=4$ mice per dose. d. Variability of the injection procedure, reproducibility or human error expressed as 


\section{WILEY-VCH}

the dose calculated for each mouse and the CV of their mean. e. Scatter plot representing the Expected vs Obtained dose values. Each dot in the plot signifies an individual calculated value for each mouse, time point and dose. Colored area within dotted lines, 95\% confidence interval. The linear correlation was tested by ANOVA $(p<0.001)$. The significance of the equation slope was statistically proven by a linear regression $t$-test. f. Accuracy of results for each particular dose expressed as error percentage.

Genipin is incorporated into hydrogel-based immunoisolation devices as a quantitative imaging probe. Increasing doses of microcapsules are injected subcutaneously in mice, obtaining strong, stable fluorescence with good linearity of signal to microcapsule dose over several weeks. This allows immediate assessment of the actual injected dose and monitoring of its position over time, thereby enhancing the efficacy and biosafety of the therapy.

\section{Keywords}

Genipin, quantitative imaging, biosafety, immunoisolation devices, hydrogels

E. Santos-Vizcaino, H. Haley, A. Gonzalez-Pujana, G. Orive, R. M. Hernandez, G. D. Luker*, J. L. Pedraz*

\section{Monitoring Implantable Immunoisolation Devices with Intrinsic Fluorescence of Genipin}
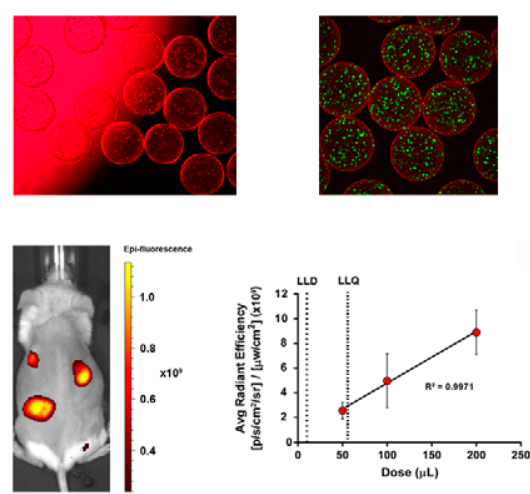
Copyright WILEY-VCH Verlag GmbH \& Co. KGaA, 69469 Weinheim, Germany, 2016.

Supporting Information

Monitoring Implantable Immunoisolation Devices with Intrinsic Fluorescence of Genipin

Edorta Santos-Vizcaino, Henry Haley, Ainhoa Gonzalez-Pujana, Gorka Orive, Rosa Maria Hernandez, Gary D. Luker*, Jose Luis Pedraz*

\section{Experimental Section}

a. Cell culture

Balb/c mouse-derived D1-MSCs (ATCC CRL12424) genetically engineered to secrete hEPO (D1-MSC-hEPO) were maintained in complete medium, consisting of: Dulbecco's modified Eagle medium (DMEM) (ATCC 30-2002) supplemented with 10\% fetal bovine serum (FBS) and $1 \%$ penicillin/streptomycin $(\mathrm{P} / \mathrm{S})$. Cells were seeded in $\mathrm{T}$-flasks, grown at $37^{\circ} \mathrm{C}$ in a $5 \%$

This article is protected by copyright. All rights reserved. 


\section{WILEY-VCH}

$\mathrm{CO}_{2} / 95 \%$ atmosphere and passaged every 2-3 days. All reagents were purchased from Gibco (Life Technologies).

\section{b. Generation of D1-MSC-hEPO clone}

D1-MSC-hEPO clone was generated in the University Clinic of Navarra (CUN) with kind technical assistance from Dr Prosper's group. Cells were transduced with the pSIN-EF2-EpoPur lentiviral vector following the same procedure previously described by our group. ${ }^{[25]}$ Briefly, the 293T packaging cell line (Clontech, Lenti-X ${ }^{\mathrm{TM}}$ 293T Cell Line, Cat. \#632180) was transfected with $9 \mu \mathrm{g}$ pSIN-EF2-Epo-Pur purified lentiviral vector, $3 \mu \mathrm{g}$ of pMD2.G (Addgene plasmid 12259) and $6 \mu \mathrm{g}$ of psPAX2 (Addgene plasmid 12260). Transfection was carried out by using $45 \mu \mathrm{L}$ of lipofectamine ${ }^{\circledR} 2000$ (Invitrogen, Carlsbad,CA,USA) according to manufacturer's protocol. 72 hours after transfection, virus-containing culture supernatant from packaging cells together with $4 \mu \mathrm{g} / \mathrm{mL}$ of Polybrene (hexadimethrine bromide; Sigma, Cat. \#H9268) were added to the D1-MSC cells. Successfully transduced cells were selected in complete medium containing $12.5 \mu \mathrm{g} / \mathrm{mL}$ of puromycin for 1 month.

\section{c. Encapsulation}




\section{WILEY-VCH}

Microcapsule matrices (beads) were produced by means of an electrostatic droplet generator (Nisco, Var V1). Briefly, D1-MSC-hEPO cells were harvested from T-175 culture flasks using trypsin-EDTA (ThermoFisher Scientific, Cat. \#25200056), filtered through a $40 \mu \mathrm{m}$ pore mesh, centrifuged at $300 \mathrm{~g}$ and gently resuspended with a spatula in $1.5 \%$ sodium alginate (ProNova UP LVG, NovaMatrix, Cat. \#4200006) at $5 \times 10^{6}$ cells/mL density. When empty capsules were used, this step was skipped. Thus, $1.5 \%$ alginate solution, with or without cells, was extruded through a $0.35 \mathrm{~mm}$ needle using a single syringe infusion pump (KD Scientific, Cat. \#78-9100) at a $5.9 \mathrm{~mL} / \mathrm{h}$ flow rate. Electric potential difference between the needle tip and the gelling solution was kept at $8 \mathrm{~V}$. Dropped beads were collected in a 100 $\mathrm{mM} \mathrm{CaCl} 2$ gelling solution and maintained in agitation for 10 minutes to ensure complete ionic gelation. Beads were then washed and coated according to the following microcapsule design:

AP: Classic alginate-poly-l-lysine microcapsules were obtained by suspending alginate beads in $0.05 \%$ PLL (Sigma-Aldrich, Cat. \#P7890) solution for 5 minutes and subsequent washing with DPBS w/Ca ${ }^{++}$and $\mathrm{Mg}^{++}$(Lonza, Cat. \#BE17-513F) to remove non-adhered polycation.

APG: AP microcapsules were covalently cross-linked with $0.1 \%$ genipin (Wako, Cat. \#07803021) for 5 minutes to form APG microcapsules, and then washed to remove unreacted cross-linker molecules.

APP: AP microcapsules were coated with a second coating of PLL following the same procedure described above ( $0.05 \%$ PLL and 5 min exposure). 


\section{WILEY-VCH}

APGP: APG microcapsules with a second coating of PLL were obtained by suspending APG microcapsules in $0.05 \%$ PLL solution for 5 min followed by washing.

GDP: APGP microcapsules were subjected to a second cross-linking reaction following the same conditions as above ( $0.1 \%$ genipin and 5 min exposure) to covalently bind the external PLL coating. Subsequently, unreacted genipin was washed away.

All groups showed a quite similar particle-size ( $\mathrm{H} 410 \mu \mathrm{m}$ diameter) with narrow distribution $(\mathrm{CV}<3 \%)($ Figure S5).

Considering electrostatic forces mainly mediate interactions between alginate matrix and PLL coating, we also discarded any possible effect of genipin cross-linking on the PLL net positive charge (See $d$. Zeta potential of PLL chains in solution). Thus, we proved that genipin crosslinking affects the positive charges of PLL chains only at $6 \mathrm{~h}$ (increasing) and $24 \mathrm{~h}$ (reducing) time points of the reaction, obtaining non-significant differences against PLL control upon completion of cross-linking (48 h) (Figure S6).

All solutions described here were within physiological $\mathrm{pH}$ and osmolarity ranges as recently described by our group. ${ }^{[26]}$ The whole encapsulation process was carried out under aseptic conditions and at room temperature. All resulting microcapsules were cultured in complete medium at $37^{\circ} \mathrm{C}$ in a $5 \% \mathrm{CO} 2 / 95 \%$ air atmosphere.

\section{d. Zeta potential of PLL chains in solution}

Zeta potential of PLL chains in solution was measured by Laser Doppler Velocimetry (LDV) using a Zetasizer Nano ZS (Malvern Instrument). PLL and genipin were dissolved in 


\section{WILEY-VCH}

deionized water at a final concentration of $0.05 \%$ and $0.1 \%$ respectively. As a control PLL $(0.05 \%)$ without genipin was used. Samples $(100 \mu \mathrm{L})$ were diluted with $0.1 \mathrm{mM} \mathrm{NaCl}(900$ $\mu \mathrm{L}$ ) and placed in a folded capillary cell for analysis. Zeta potential obtained from electrophorectic mobility was supported using the Smoluchowski approximation. Only data that passed the quality criteria stablished by the sofware DTS 5.0 were included in the analysis. Data is represented as mean $\pm \mathrm{SD}$ of three independent samples.

\section{e. Optimization of genipin cross-linking procedure}

To determine optimum conditions for the cross-linking procedure, cells enclosed within AP microcapsules were subjected to increasing concentrations of genipin and exposure times. The final cross-linking procedure protocol was established on the basis of the highest fluorescence signal intensity obtained without affecting cell viability when compared to cells encapsulated in non-cross-linked AP microcapsules.

\section{f. Assessment of fluorescence signal intensity}

Fluorescence intensity was analyzed qualitatively by either epi-fluorescence microscopy (overall view) or confocal fluorescence microscopy (z-sectioning). For confocal images, profile lines and 3D surface plots of the equatorial section were built with Image $J 1.49 \mathrm{i}$ (Wayne Rasband, National Institutes of Health, USA). For quantitative data, 65 microcapsules-containing $100 \mu \mathrm{L}$ of DPBS solution were pipetted per well $(n=5)$ into a 96 well black plate. Fluorescence signal intensity was measured at $590 \mathrm{~nm}$ excitation and $630 \mathrm{~nm}$ emission wavelengths using a Tecan Infinite M200 plate-reader based on excitation/emission spectra obtained previously (Supplementary Fig. 1) with GDP capsules under the same 


\section{WILEY-VCH}

conditions. For emission spectrum, GDP capsules were excited at $514 \mathrm{~nm}$ and emitted fluorescence was recorded from 550 to $700 \mathrm{~nm}$. As for excitation spectrum, 500-650 nm range was scanned while reading the fluorescence emitted at $690 \mathrm{~nm}$. In both cases, a step size of $2 \mathrm{~nm}$ was used. Obtained data are the mean of 4 replicates.

\section{g. Cell viability/Metabolic activity}

Metabolic activity of enclosed cells was assessed using CCK-8 cell viability kit (Sigma, Cat. \#96992). Approximately 15,000 cells/well were plated in a 96 well plate. Then, $10 \mu \mathrm{L} /$ well of CCK-8 were added. After 3 hours of incubation at $37^{\circ} \mathrm{C}$, the plate was read at $450 \mathrm{~nm}$ to measure colour development. Measured values were corrected with the reference wavelength at $690 \mathrm{~nm}$ and normalized against the mean value of 5 blank wells (medium). Results are expressed as the mean of 5 independent samples \pm SD for every assay condition or study group.

\section{h. Live/Dead assay}

Encapsulated cells were dyed with the calcein-AM/ethidium homodimer-1 fluorescent probes

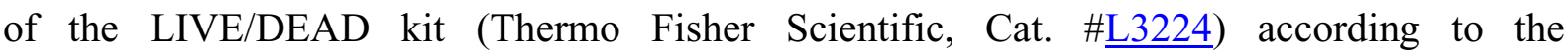
manufacturer's indications. After $30 \mathrm{~min}$ of incubation at room temperature and protection from light, fluorescence micrographs were taken using a confocal fluorescence microscope (Leica TCS SP2 AOBS Spectral Confocal Scanner mounted on a Leica DM IRE2 inverted fluorescent microscope). The obtained micrographs were mounted in a z-stack using Image $J$. 


\section{WILEY-VCH}

\section{i. Measurement of hEPO secretion}

Secretion of hEPO was measured in H135,000 encapsulated cells/well placed in 12 well plates with $1 \mathrm{~mL}$ medium. After a 24 hour release period, cell supernatants were assayed for hEPO secretion using the Quantikine IVD Human Erythropoietin ELISA Kit (R\&D Systems, Cat. \#DEP00). Standards and samples were run in duplicate as specified in the kit, and all results were expressed as the mean $+\mathrm{SD}$ of 3 independent experiments per study group.

\section{j. In vivo imaging}

To determine stability of imaging signal from genipin microcapsules in vivo, we implanted 50, 100 or $200 \mu \mathrm{L}$ of microcapsules subcutaneously into 15 -week-old female NSG mice $(\mathrm{n}=4$ per group). The University of Michigan IACUC approved all animal procedures. We implanted microcapsules through a $18 \mathrm{G}$ x 11/4 NIPRO SAFELET CATH (obtained from University of Michigan Hospital Pharmacy) into the dorsal region of each mouse. We imaged fluorescence from implanted microcapsules using $570 \mathrm{~nm}$ excitation and a 620/20 emission filter on an IVIS Spectrum (Perkin Elmer) as described previously. ${ }^{[27]}$ To quantify imaging data, we measured radiant efficiency in defined regions-of-interest at each time point and then calculated mean values $\pm \mathrm{SD}$.

\section{k. Analysis of in vivo imaging data}

Background signal mean and SD were calculated from blank zones in mice at different time points ( 4 mice and 4 time points, $n=16$ ). Lower limit of detection (LLD) and lower limit of quantification (LLQ) were calculated as follows: 
$\mathrm{LLD}=($ mean of background negative control radiant efficiency $)+3 \times$ (SD of background negative control radiant efficiency).

$\mathrm{LLQ}=$ (mean of background negative control radiant efficiency) $+10 \mathrm{x}$ (SD of background negative control radiant efficiency).

Variability of the technique, namely repeatability or instrumental error, was determined according to the mean of the coefficient of variation (CV) obtained from different measurements of the same dose at different time points $(1,14,21$ and 35 days) for each mouse.

Variability of the injection procedure, reproducibility or human error (i.e. dose preparing and administration of microcapsules) was resolved for each dose. The mean obtained from the same measurement along the four time points approximates the actual administered dose for each mouse, reducing data dispersion derived from instrumental error (repeatability). Then, the grand mean (mean of the means) of the four mice was calculated, from which the CV was considered as indicator of the injection procedure variability.

The accuracy of results for each particular dose denotes the proximity of obtained values from expected ones, regardless of the dispersion resulting from either the instrument or human error. The mean from the same measurement along the four time points, whereby it is possible to obtain the nearest value of the actual administered dose for each mouse, was first calculated to minimize data dispersion derived from instrumental error (repeatability). Then, the grand mean (mean of the means) of the four mice was calculated to reduce variability caused by 


\section{WILEY-VCH}

human error. The resultant value was subtracted from the expected value and expressed as error percentage.

\section{l. Data analysis and statistics}

Results are shown as mean \pm SD for line and dot graphs. The normal distribution of the data and homoscedasticity were checked by Shapiro-Wilk and Levene tests respectively. An unpaired, two-tailed $t$-test was used to detect statistical significances between two groups, while one-way ANOVA was used for multiple comparisons. In this case, Bonferroni or Tamhane post-hoc tests were applied depending on the obtained homogeneity of variances. When non-normally distributed data were observed, a Mann-Whitney non-parametric test was applied. Differences between means obtained within the same group along the time were probed using a paired, two-tailed $t$-test. Each figure caption specifies the sample size $(n)$ and the statistical test used for each assay. $p<0.05$ was considered statistically significant. All statistical computations were performed using SPSS 22 (IBM SPSS, Chicago, IL). ${ }^{*}, p<$ $0.05 ; * *, p<0.01 ; * * *, p<0.001$. 


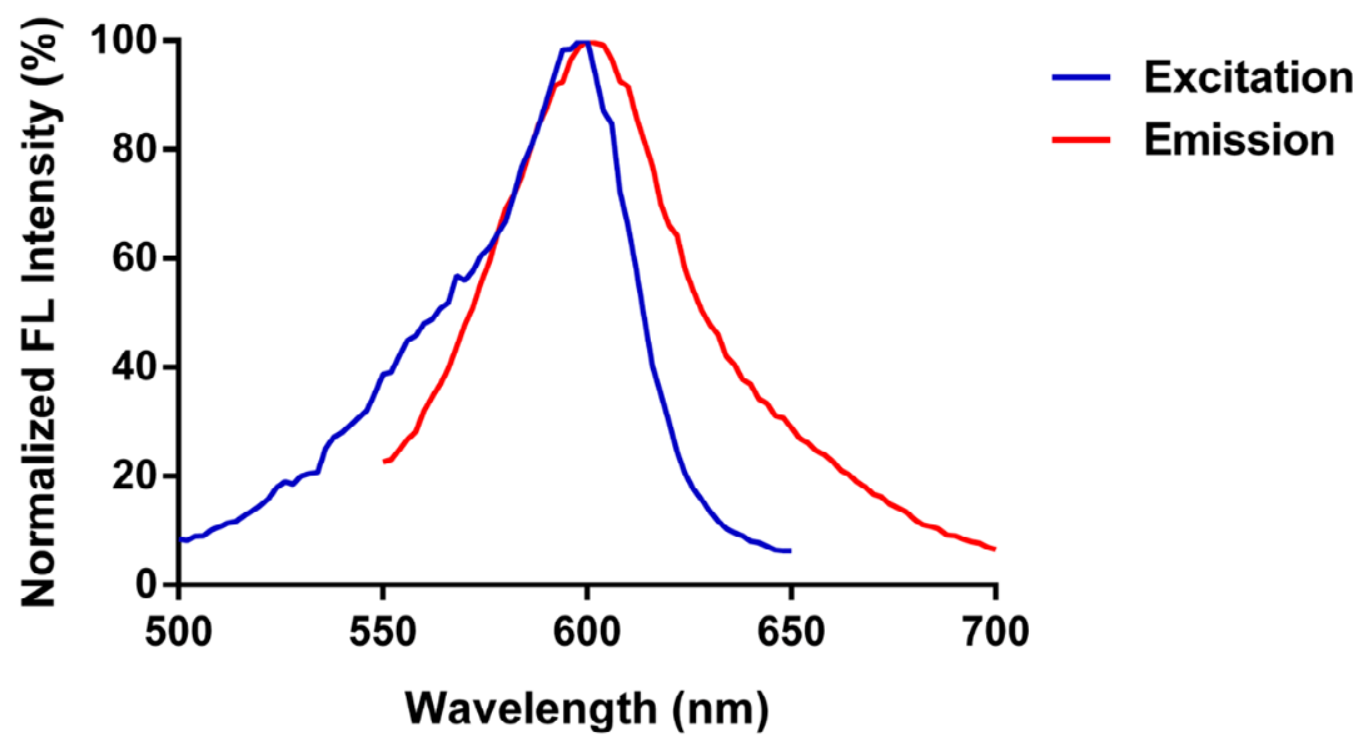

Figure S1. Excitation and emission spectra of genipin cross-linked microcapsules. Emission spectrum, Ex: 514 nm / Em: 550-700 nm. Excitation spectrum, Ex: 500-650 nm / Em: 690 $\mathrm{nm}$. Step size, $2 \mathrm{~nm}$. Obtained data are the mean of 4 replicates.

This article is protected by copyright. All rights reserved. 
a

N.S.

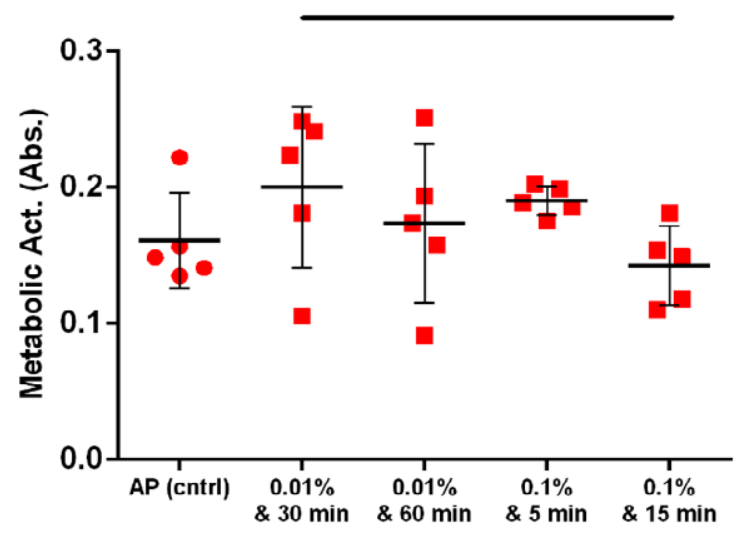

b

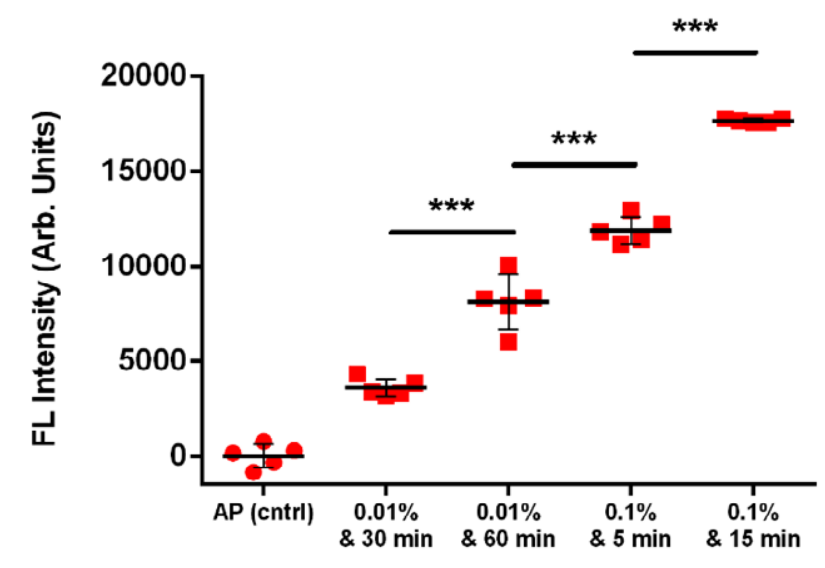

Figure S2. D1-MSC-EPO encapsulated in AP microcapsules and subjected to the genipin cross-linking protocols selected in Figure 1b,c. Non-cross-linked AP group was used as control. a, Metabolic activity of encapsulated cells after genipin cross-linking. Error bars, mean \pm SD. N.S. non-significant differences; One-way ANOVA with Bonferroni multiple comparison correction, $n=5$ per condition. $\mathbf{b}$, Fluorescence intensity of AP microcapsules after genipin cross-linking. Error bars, mean $\pm \mathrm{SD}$. ${ }^{* * *}, p<0.001$; One-way ANOVA with Bonferroni multiple comparison correction, $n=5$ samples per condition. 


\section{WILEY-VCH}

a

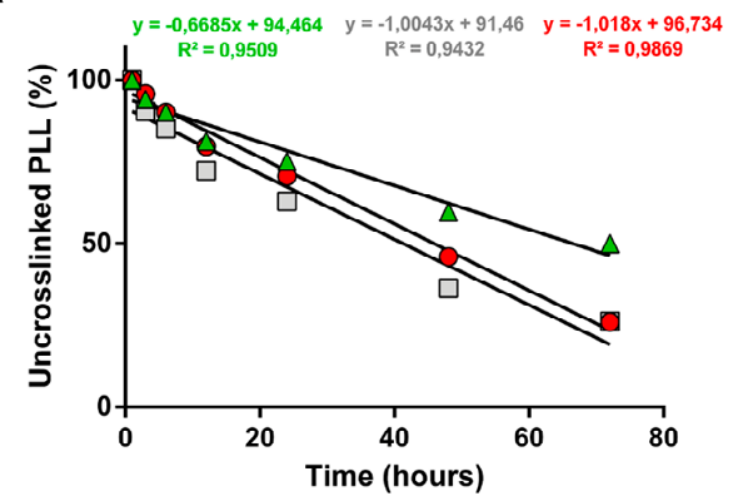

b

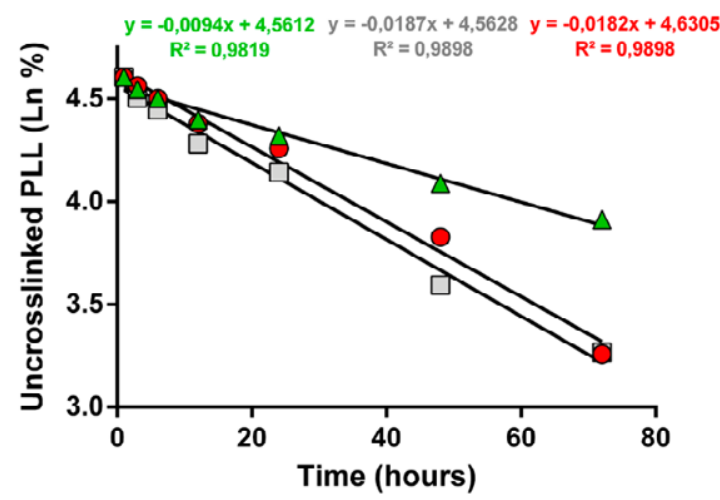

Figure S3. Cross-linking reaction kinetics of GDP microcapsules and the intermediate control groups $(n=4)$. Linear least squares fitting method to demonstrate zero order (a) or first order $(\mathbf{b})$ reactions. 


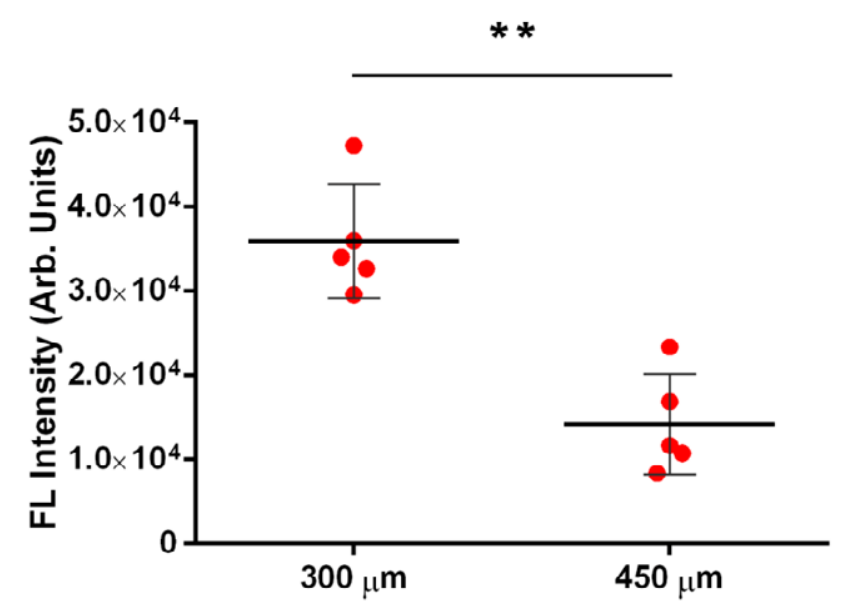

Figure S4. Diameter size-dependent fluorescence intensity in GDP microcapsules. Error bars, mean \pm SD. ${ }^{* *}, p<0.01$; Unpaired, two-tailed $t$-test, $n=5$ samples per size.

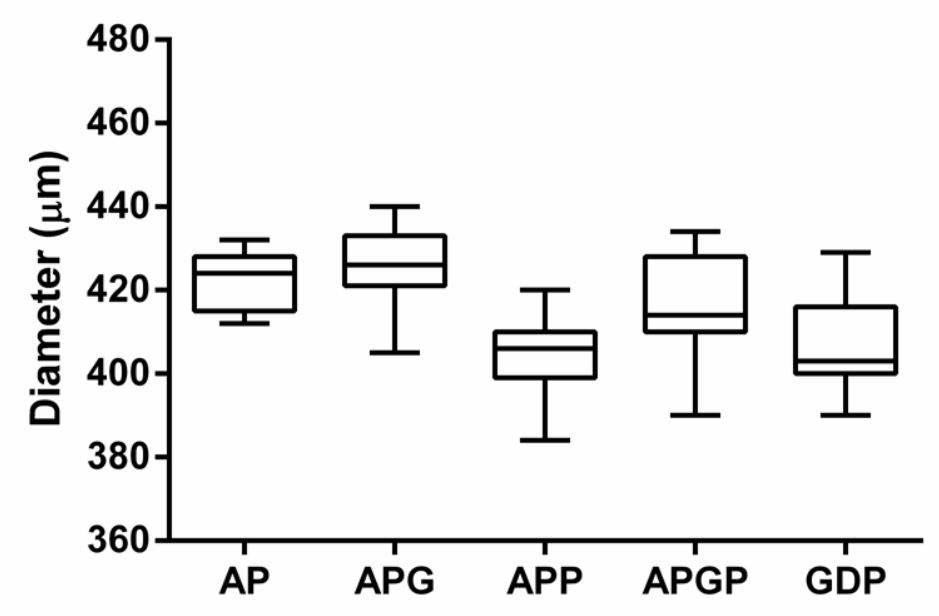

Figure S5. Diameters of GDP microcapsules and intermediate control groups $(n=15$ capsules per group). Boxes, interquartile range (Q1-Q3); Central point, median; Whiskers, max. and min. values.

This article is protected by copyright. All rights reserved. 


\section{WILEY-VCH}

This article is protected by copyright. All rights reserved. 


\section{WILEY-VCH}

a

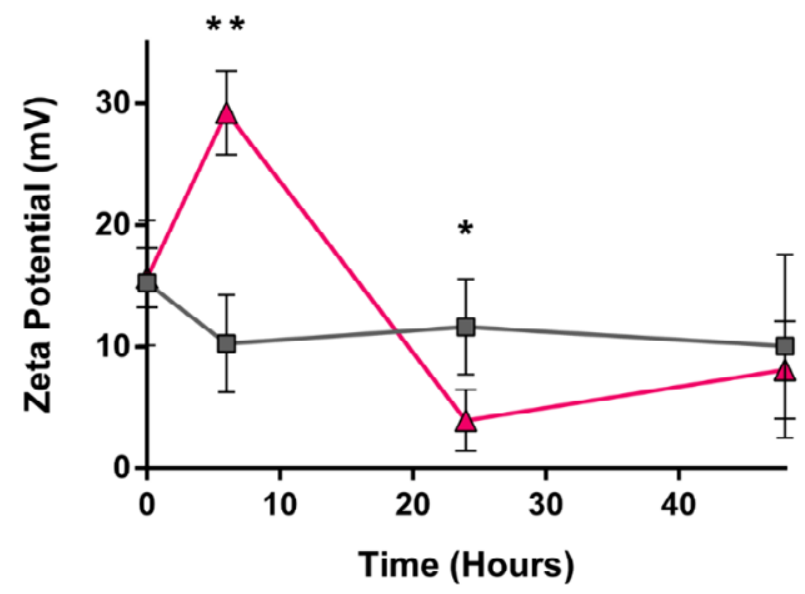

b

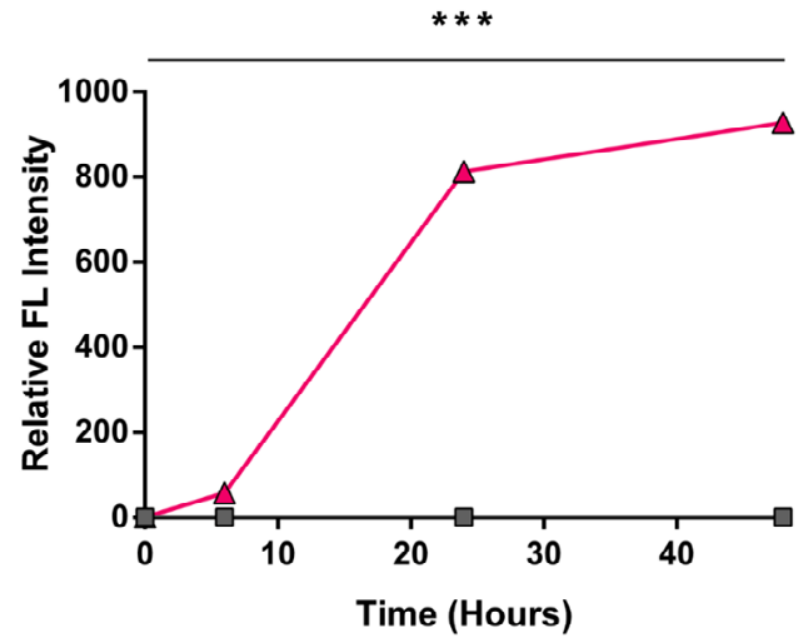

Figure S6. a, Zeta potential of PLL chains in solution throughout genipin cross-linking reaction time-course. Error bars, mean $\pm \mathrm{SD} .{ }^{*}, p<0.05$; ${ }^{* *}, p<0.01$; Unpaired, two-tailed $t$ test, $n=3$ per solution. $\mathbf{b}$, Monitoring of the cross-linking reaction by means of genipin emitted fluorescence. Error bars, mean $\pm \mathrm{SD}$ (smaller than markers). ${ }^{* * *}, p<0.001$; Unpaired, two-tailed $t$-test, $n=3$ per solution.

Video S1. 3D reconstruction using a stack of confocal images of GDP capsules to show its morphology. 


\section{WILEY-VCH}

Video S2. 3D reconstruction displaying fully viable cells (green) within GDP capsules (red). $3 \mathrm{D}$ view from a stack of confocal images.

Supplementary References

[25] H. Gurruchaga, J. Ciriza, L. Saenz Del Burgo, J. R. Rodriguez-Madoz, E. Santos, F. Prosper, R. M. Hernández, G. Orive, J. L. Pedraz, Int. J. Pharm. 2015, 485, 15.

[26] A. Gonzalez-Pujana, A. Rementeria, F.J. Blanco, M. Igartua, J.L. Pedraz, E. SantosVizcaino, R.M. Hernandez. Drug Deliv. 2017, 24(1), 1654.

[27] K. E. Luker, P. Pata, I. I. Shemiakina, A. Pereverzeva, A. C. Stacer, D. S. Shcherbo, V. Z. Pletnev, M. Skolnaja, K. A. Lukyanov, G. D. Luker, I. Pata, D. M. Chudakov, Sci. Rep. 2015, 5, 10332. 

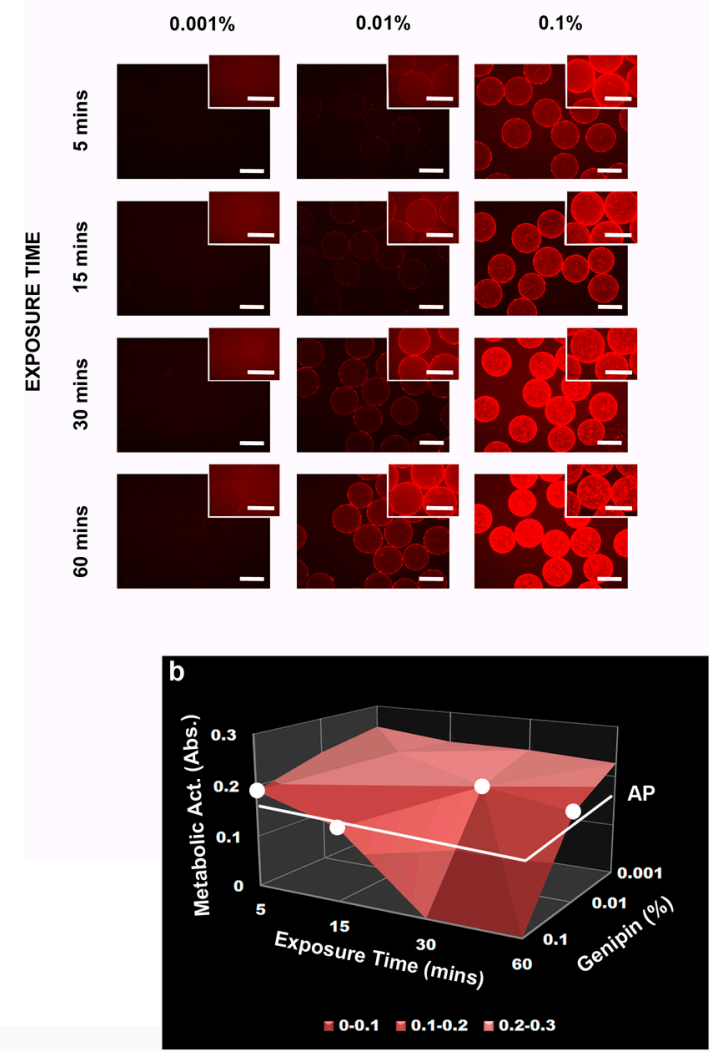

d

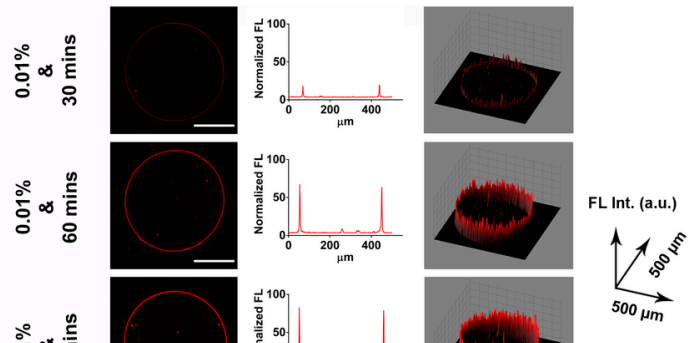

$\stackrel{\circ}{\circ} \infty \frac{n}{\frac{0}{E}}$
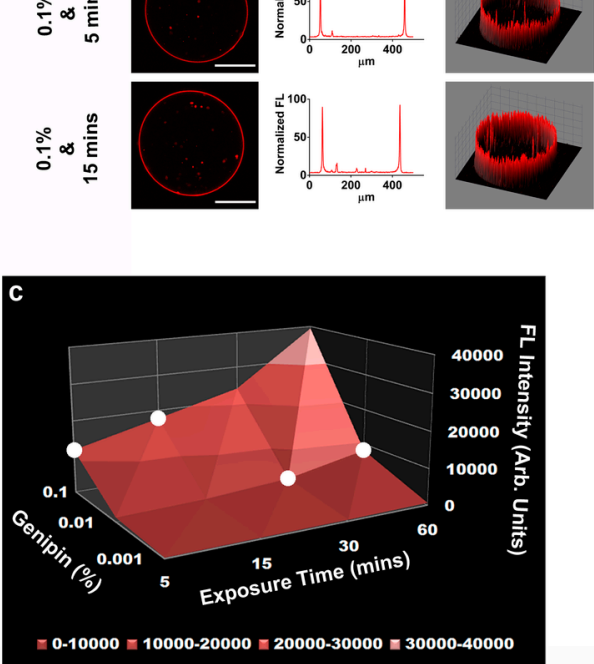

Figure 1.tif

This article is protected by copyright. All rights reserved. 
a
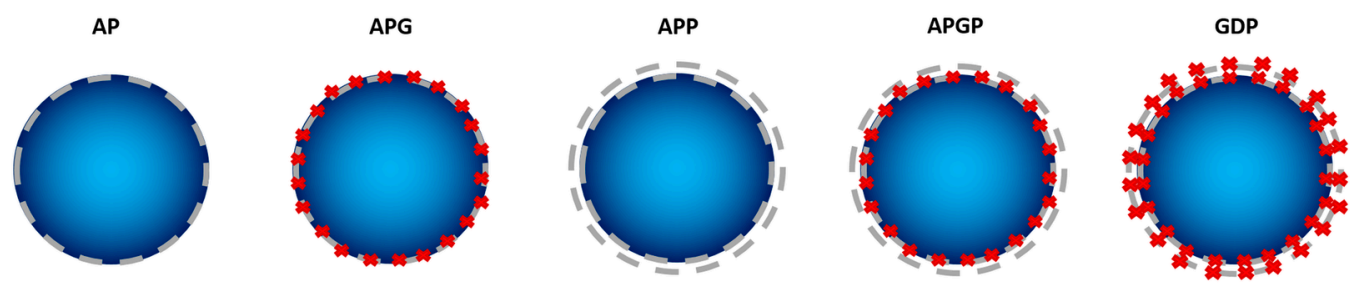

Alginate matrix

Poly-L-lysine coating

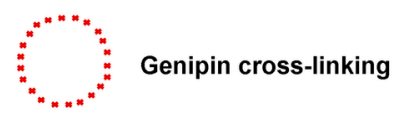

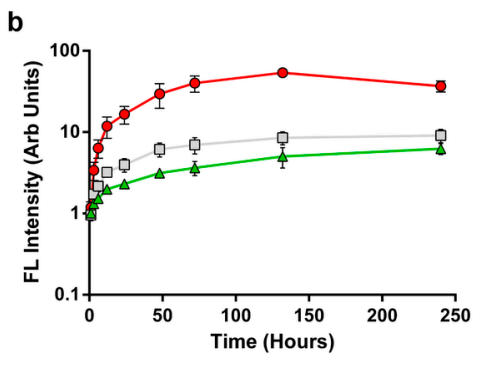

$\triangle$ APG $\square$ APGP $\rightarrow$ GDP

d
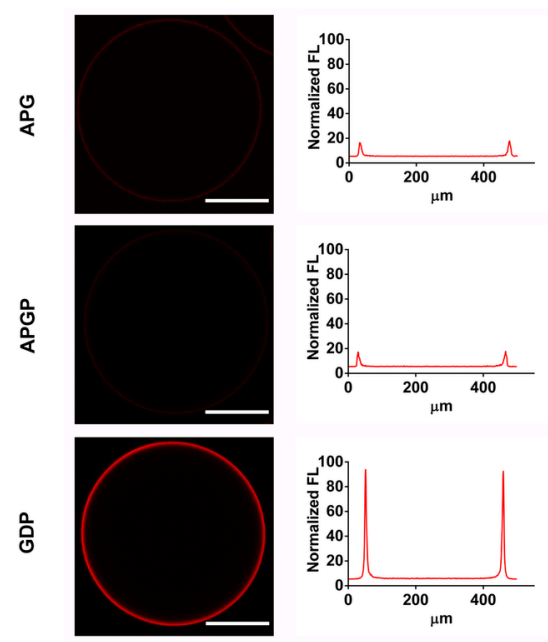
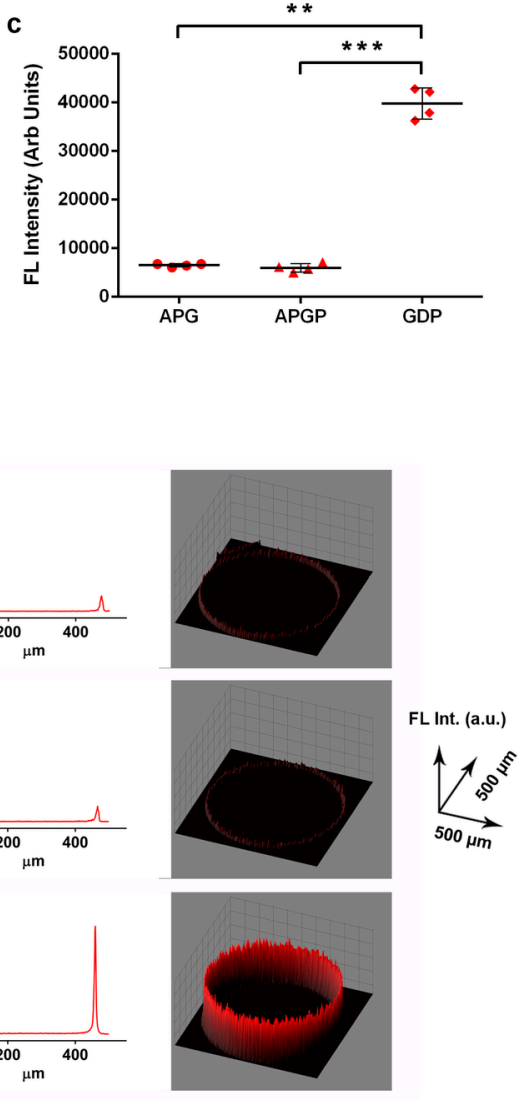

Figure 2.tif

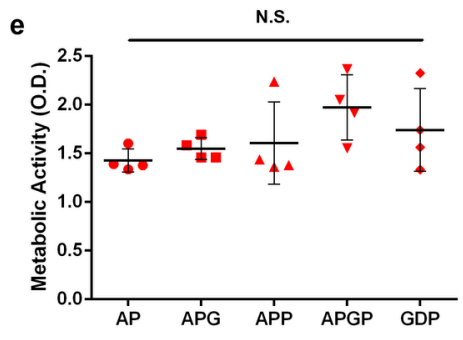

f
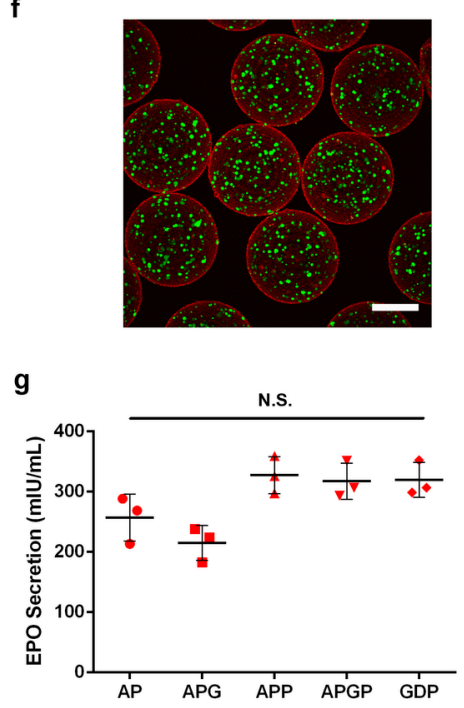


\section{b}

a

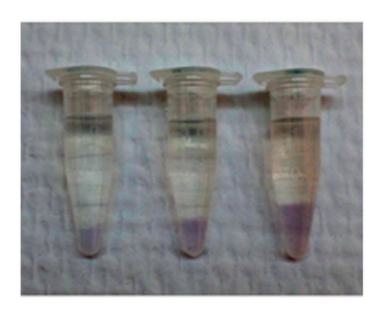

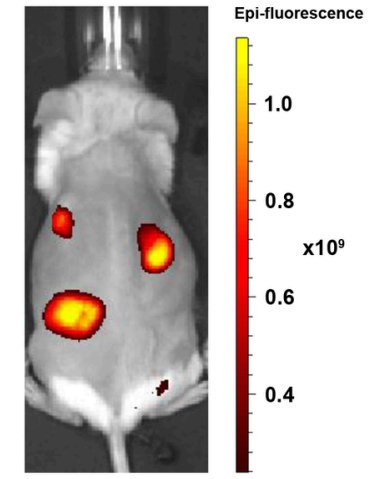

C

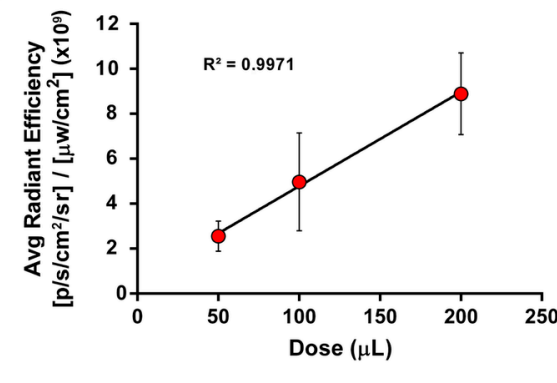

d

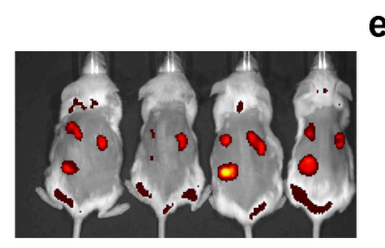

e

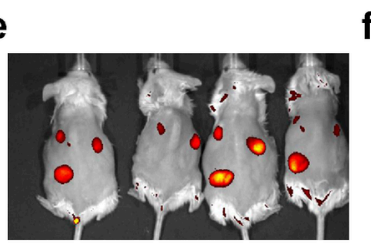

f

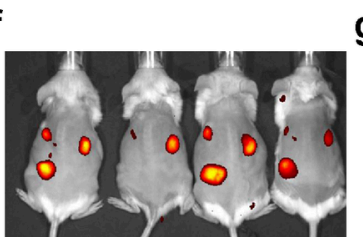

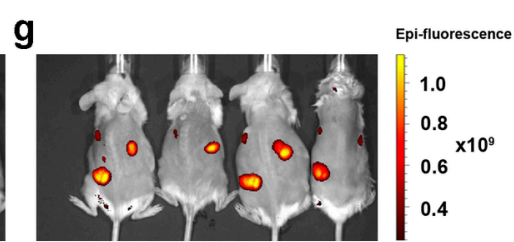

h

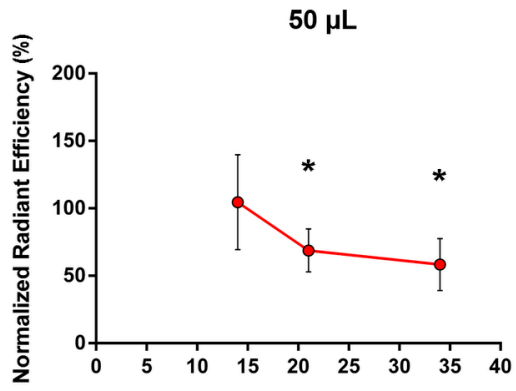

$100 \mu \mathrm{L}$
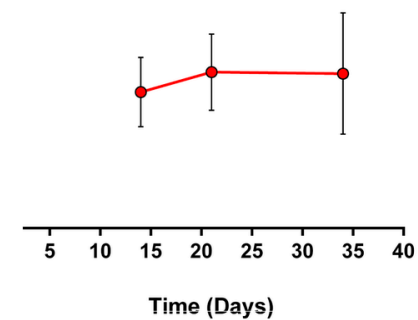

$200 \mu \mathrm{L}$
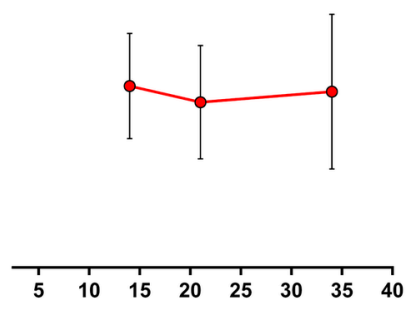

Figure 3.tif

This article is protected by copyright. All rights reserved. 
a

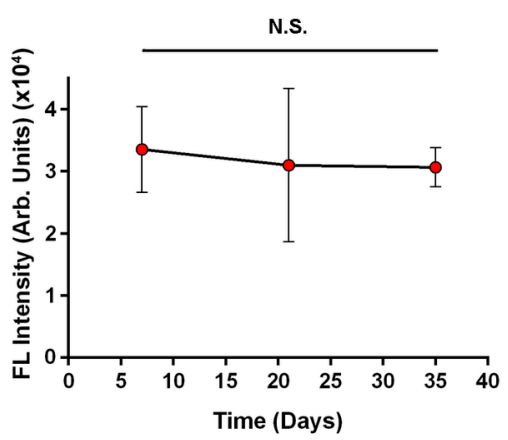

C

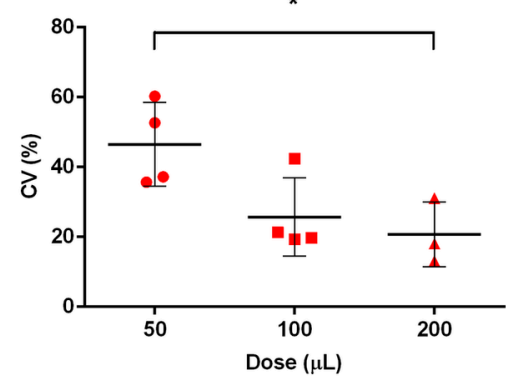

e

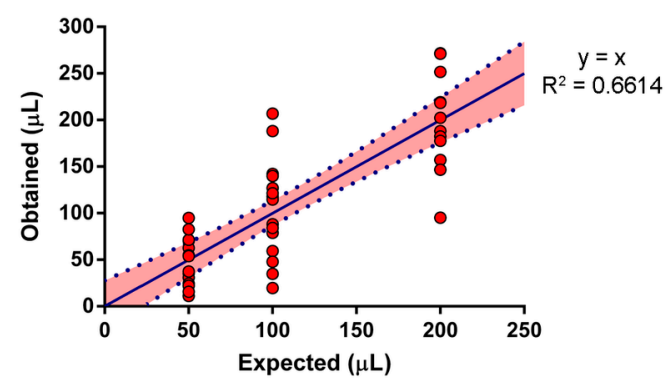

b

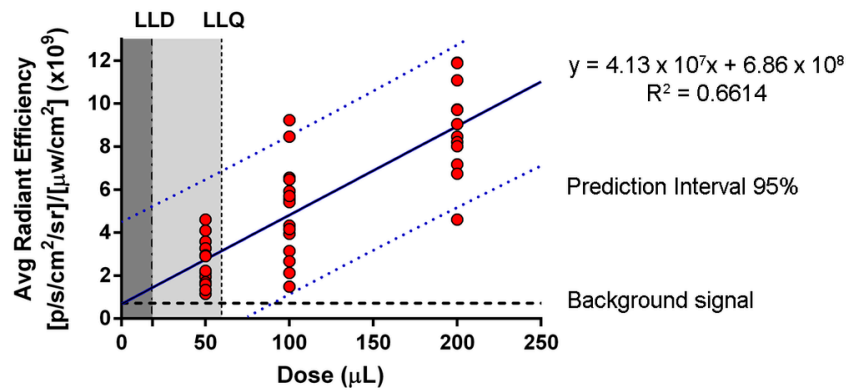

$a=41,339,506(p<0.001)$ $b=685,622,093$

d

\begin{tabular}{lccc}
\hline Human error & $\mathbf{5 0}$ & $\mathbf{1 0 0}$ & $\mathbf{2 0 0}$ \\
\hline Mouse 1 Dose $(\boldsymbol{\mu L})$ & 65.8 & 99.9 & 171.1 \\
Mouse 2 Dose $(\boldsymbol{\mu L})$ & 30.3 & 104.9 & \\
Mouse 3 Dose $(\boldsymbol{L L})$ & 62.0 & 169.3 & 249.1 \\
Mouse 4 Dose $(\boldsymbol{\mu L})$ & 32.2 & 40.5 & 174.9 \\
\hline \hline CV $(\%)$ & 39.8 & 50.8 & 22.2 \\
\hline
\end{tabular}

\section{f}

\begin{tabular}{lccc}
\hline Accuracy & & & \\
\hline Expected Val. $(\mu \mathrm{L})$ & 50 & 100 & 200 \\
Obtained Val. (mean) $(\mu \mathrm{L})$ & 47.6 & 103.6 & 198.4 \\
\hline \hline Error of measurement $(\%)$ & -4.82 & 3.62 & -0.80 \\
\hline
\end{tabular}

$a=1(p<0.001)$

$b=-0.00000000215$

Figure 4.tif

This article is protected by copyright. All rights reserved. 

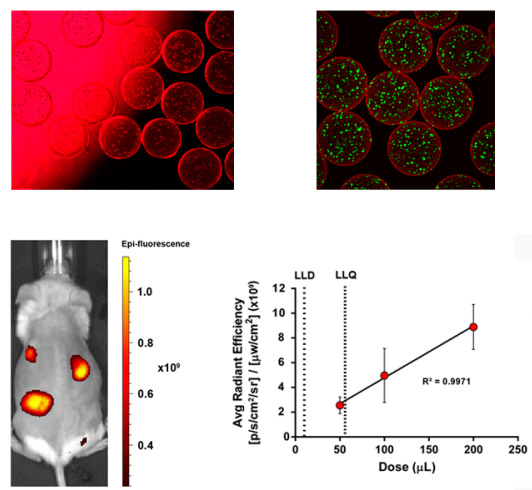

Graphical Abstract.tif

This article is protected by copyright. All rights reserved. 